\title{
Relação mãe-filho(a) em bebés transportados junto ao peito e em bebés transportados em carrinhos
}

\author{
Carina Santa Bárbara ${ }^{1}$, Marina Fuertes ${ }^{1,2}$ \& Olívia Carvalho ${ }^{3}$ \\ ${ }^{1}$ Escola Superior de Educação de Lisboa, Instituto Politécnico de Lisboa, Lisboa \\ 2 Universidade do Porto, Centro de Psicologia, Porto \\ ${ }^{3}$ Universidade Portucalense Infante D. Henrique, Porto
}

\begin{abstract}
Resumo: Sabendo que o contacto físico é importante na relação mãe-filho(a), procurámos comparar a qualidade da interação mãe-filho(a) em dois grupos de estudo: Grupo 1) 20 bebés transportados junto ao peito das suas mães e Grupo 2) 20 bebés transportados em carrinhos ou outros meios auxiliares. Os dois grupos foram emparelhados por idade gestacional, peso gestacional, por idade da criança, sexo da criança, idades dos pais, nível socioeconómico, e nacionalidade. Os bebés tinham entre 6 e 36 meses (20 meninas, 13 primíparos) e não apresentavam problemas de desenvolvimento. A qualidade da interação mãe-filho foi avaliada em jogo livre através das escalas CARE-Index e MINDS. Comparativamente ao grupo 1, o grupo 2 apresenta melhor qualidade interativa (médias superiores de sensibilidade materna e de cooperação infantil). Estes dados corroboram a premissa de que mais importante do que proximidade é a forma como ela é estabelecida, que contribuirá para a qualidade da relação mãe-filho(a).
\end{abstract}

Palavras-chave: Transporte do bebé; contacto; proximidade física; qualidade interativa mãe-filho(a).

Mother-Infant Relationship in maternal baby wearing and trolleys transport: Taking that maternal touch is a key factor in mother-infant relationships, we aim to compare the quality of mother-infant interaction in two groups: Group 1) 20 infants carried next to their mothers' bodies and Group 2) 20 infants who were carried in trolleys or other aids for transportation. Infants were between 6 and 36 months of age (20 girls, 13 primiparous) without developmental problems. Both groups were paired by gestational age, birth weight, children sex, children age, parents' age, SES and nationality. The quality of the mother-infant interaction was evaluated in a free play situation with CARE-Index and MINDS scales. Our findings indicate that Group 1, in comparison with group 2, had lower dyadic quality. These findings support the idea that more than body closeness, it is the quality of interaction where such closeness occurs, that contributes to the quality of mother-infant relationships.

Keywords: Mother-child bonding; dyadic relationship; physical proximity; contact; mother-infant relationship.

Em sociedades industrializadas e desenvolvidas, o transporte corporal dos bebés, como alternativa ao carrinho, parece estar a ser reintroduzido tendo surgido no mercado novas ofertas como panos, slings e marsúpios. Nos EUA, embora $99 \%$ dos pais tenha carrinho e $17 \%$ usa, adicionalmente, marsupiais ou outros meios de transporte corporal (Blois, 2005). Porém, pouco se sabe sobre as vantagens deste tipo de transporte para a saúde e desenvolvimento do bebé, para a saúde dos pais e para a relação entre os pais e os bebés. É previsível que o transporte junto ao corpo tenha tido origem nas populações nómadas, facilitando as deslocações das mães, embora o uso de meios auxiliares de transporte tenha sido quase completamente extinto nas sociedades modernizadas. Assim, questionamo-nos como será reintroduzir na sociedade portuguesa o transporte junto ao corpo? E quais os contributos para a interação mãe-filho(a)? Na verdade, a sociedade portuguesa (especialmente a sociedade urbana) recorre a meios auxiliares de transporte nos últimos 50 anos, distanciando-se culturalmente do transporte junto ao corpo. Contudo, algumas famílias optam pela sua reintrodução mesmo não se tratando de um hábito familiar ou cultural. Procuraremos estudar a qualidade da interação mãe-filho(a), em díades portuguesas com transporte corporal (junto ao peito ou às costas) e comparar com díades no qual os bebés são transportados em carrinhos. Queremos, igualmente, saber que aspetos específicos da relação mãe-filho estão associados ao transporte corporal, nomeadamente, em termos de resposta facial, vocal e afetiva, sincronia, contingência e estabelecimento lúdico.

${ }^{1}$ Morada para correspondência: Marina Fuertes, ESELX, Campus do IPL, Benfica. E-mail: marinaf@eselx.ipl.pt. Esta investigação é financiada pela Fundação para a Ciência e Tecnologia (PTDC/MHC-PED/1424/2014). 


\section{Vinculação e Proximidade física}

Segundo Bowlby (1969), o estabelecimento de relações de vinculação é, em si, um fenómeno normativo e universal na espécie humana. Contudo, a qualidade da vinculação deve ser entendida de forma sistémica, resultante de múltiplos fatores e interações (Cassidy, 2008).

A qualidade da vinculação decorre do conceito de base segura. A maioria das crianças, recorre à sua figura de vinculação como uma base segura, ou seja, quando a criança perceciona "perigo" (e.g., presença de estranhos, dor medo, ausência materna) procura a proteção materna (aproximando e tentando obter o contacto). Deste modo, a forma como a figura materna responde à procura de proximidade física e contacto por parte da criança é determinante da qualidade da relação (Ainsworth, Blehar, Waters \& Wall, 1978).

Com efeito, a forma como as crianças regulam a proximidade e o toque com a figura de vinculação é distinta nos vários tipos de vinculação. Ainsworth e colaboradores (1978) conceptualizaram a qualidade dos vínculos em três grandes padrões: o B (seguro), o A (inseguro-evitante) e o C (inseguroresistente/ambivalente). Na vinculação insegura-evitante a criança explora o meio de forma independente, não mostrando desconforto com a ausência da mãe e evitando ativamente o contacto e a proximidade da mãe após o reencontro. A vinculação evitante tem sido associado a problemas de internalização, saúde mental e isolamento (Thompson, 2008).

0 tipo de vinculação segura é marcado por uma figura de vinculação que atua como uma base segura, para a qual a criança pode regressar após a exploração do meio. 0 regresso é vivido com interesse e, na maioria dos casos, com procura de contacto. A investigação tem identificado a vinculação segura como uma fonte de proteção e fonte de resiliência para o desenvolvimento socioemocional e para a aprendizagem (Thompson, 2008).

As crianças que demonstram uma vinculação insegura-resistente/ambivalente têm dificuldades em explorar o meio, mesmo antes da separação, procurando monitorizar a localização da figura de vinculação. Depois da reunião exibem comportamentos ambivalentes, vacilando entre a procura de proximidade e os comportamentos de resistência, ou apresentando resistência através da passividade. Este estilo de vinculação segura tem sido associado a problemas de externalização, relação com os pares, e qualidade da saúde da criança (Thompson, 2008).

Em suma, a qualidade da vinculação dependerá e resultará da procura do contacto físico, e persistência desse contacto. Alguns investigadores consideraram o contacto materno como uma variável que pode estar na origem da vinculação e que, simultaneamente, pode evoluir como expressão da sua organização (e.g., Anisfeld, Cusper, Nozyce, \& Cunningham, 1990). O contacto, o afeto e a proximidade materna podem ser o contexto relacional no qual a criança se sente segura e recebida dando inicio ao estabelecimento da vinculação

\section{Relação mãe-filho(a) e contacto físico}

Para compreender de que forma o contacto materno podia afetar a relação com a criança, constituiu-se uma linha de investigação dedicada ao tema. Por exemplo, Ferber, Feldman e Makhoul (2008) analisaram a quantidade de toque de estimulação, afetivo e instrumental materno durante atividades de rotina diária (e.g., mudanças de fraldas, alimentação), ao longo do primeiro ano de vida da criança. Os resultados indicaram que a quantidade do toque não era um fator determinante na relação na mãe-filho(a). Porém, a qualidade das experiências proporcionadas nas atividades com toque era determinante dessa qualidade, nomeadamente pela reciprocidade desencadeada e pelo envolvimento emocional proporcionado. Ora, aqui alguma investigação começa a indicar que a quantidade de toque (contacto físico) não é por si só um indicador, exceto nas díades com mães com o diagnóstico de depressão ou outras alterações somáticas (e.g., Herrera, Reissland, \& Shepherd, 2004).

A relação entre a proximidade física e a qualidade da vinculação foi, igualmente, estudada em díades em que os bebés eram transportados junto ao corpo (slings) e em díades que transportaram os bebés por meios auxiliares (e.g., carrinhos, berços). Os resultados foram muito evidentes, $83 \%$ das crianças transportadas junto ao corpo apresentaram uma relação segura face a $39 \%$ do grupo de controlo. Aos três meses de vida do bebé, nas díades com transporte corporal, as mães apresentaram comportamentos mais contingentes e recíprocos (Anisfeld, et al., 1990). Num estudo com pais que usavam os dois tipos de transporte, verificou-se que os pais e os bebés vocalizam mais e com mais sincronia quando os bebés são transportados em marsupiais em oposição aos carrinhos (Mireault, Rainville, \& Laughlin, 2018).

Em suma, os estudos não oferecem um corpo conclusivo de evidências se, por um lado, não bastará estar em contacto, por outro lado, o contacto parece beneficiar a relação. Uma prova indireta resulta dos estudos acerca eficácia das intervenções na promoção de relações seguras, que identificam que as intervenções centradas na proximidade física e no transporte corporal são eficazes em reduzir o 
distanciamento materno (e.g., Hunziker \& Barr, 1986). Destas intervenções, o método Canguru com bebés prematuros tornou-se particularmente popular pelos ganhos identificados para a saúde, bem-estar do bebé e dos pais (Fuertes, et al., 2012; Tessier, et al., 1998).

Até ao momento presente, os estudos enunciados parecem indicar que o transporte corporal é preferencial ao transporte por meios auxiliares. Contudo, os estudos escasseiam e os resultados parecem depender das singularidades do transporte corporal ou das especificidades das amostras. Por exemplo, no estudo realizado por Ferber e colegas (2008), as amostras são oriundas de famílias com baixa condição socioeconómica e sujeitas a diversos fatores de risco, em que a escolha pelo transporte corporal não é uma opção, mas sim uma necessidade. Será que os resultados se mantêm com famílias de classe médias, com maior literacia e noutras culturas? E será que para compreender o papel da variável contacto temos que compreender o contexto relacional em que ocorre (se as relações são sensíveis, positivas, afetivas, reciprocas e contingentes)?

\section{Qualidade da interação mãe-filho(a)}

Se existem diversos fatores a contribuir para a vinculação, alguns apresentam maior peso a explicar as diferenças na relação mãe-filho(a). Em todas as culturas estudadas, a sensibilidade materna ou paterna foi identificada como o maior fator explicativo da vinculação meta-analiticamente (De Wolf \& van IJzendoorn, 1997; Lucassen et al., 2011). É neste sentido que o nosso estudo se debruça sobre a relação com bebés (considerando a sensibilidade materna e cooperação infantil) transportados junto ao corpo das suas mães, seguindo o exemplo de práticas próprias da cultura africana e tanto quanto sabemos nunca antes estudado em Portugal.

A sensibilidade foi inicialmente definida por Ainsworth e colegas como a capacidade do adulto "(...) to perceive and to interpret accurately the signals and communications implicit in her infant's behavior, and given this understanding, to respond to them appropriately and promptly" (Ainsworth, Bell, \& Stayton, 1974, p. 127). Ainsworth e sua equipa (1978) iniciaram uma prolífera linha de investigação lançando-se a discussão sobre quais os fatores que contribuíam para a sensibilidade materna. Os resultados indicaram que sensibilidade materna é uma variável preditora do desenvolvimento, afetando a regulação fisiológica e emocional (e.g., Calkins \& Hill, 2007; Moore et al., 2009), a agressão entre pares (Crockenberg, Leerkes, \& Barrig Jo, 2008; Leerkes, Blankson, \& O’Brien, 2009) e o desenvolvimento cognitivo, social e emocional (Bernier, Carlson, \& Whipple, 2010; Leerkes et al., 2009; Tamis-LeMonda, Bornstein, Baumwell, \& Damast, 1996).

Mais recentemente, diversos autores (e.g., Belsky, 1999; Crittenden, 1999) defendem que a sensibilidade materna deve ser concebida como um constructo diádico, reconhecendo a importância do comportamento de ambos os elementos da díade. Crittenden (1988) define a sensibilidade materna como qualquer padrão de resposta que contribui para o bem-estar do bebé, conforto ou para o seu envolvimento na relação. Além das dimensões propostas por Ainsworth e colegas (responder às necessidades da criança), nesta perspetiva da sensibilidade materna, Crittenden (1988) inclui a sincronia, reciprocidade, estabelecimento da relação e atividade lúdica como componentes da sensibilidade materna. A corroborar esta proposta, o estudo meta-analítico de De Wolff e van IJzendoorn (1997) apresenta a mutualidade e a reciprocidade como dimensões importantes da qualidade da interação mãefilho(a). Neste sentido, Beeghly, Fuertes, Liu, Delonis, e Tronick, (2011) recorrem ao modelo de regulação mútua que prevê que os bebés e as suas mães formem um sistema diádico em que ambos corregulam as suas interações e comportamentos. 0 sucesso ou o fracasso da sua regulação mútua durante as interações sociais depende de quão clara e eficazmente cada parceiro é capaz de comunicar a sua intenção, bem como de apreender e compreender o significado e intenção do seu interlocutor. A clareza de emissão e receção é a primeira etapa de um processo que requer que os dois parceiros sejam capazes de aceitar o contributo do outro e a devolver uma resposta positiva. Neste modelo, a chave das relações está na harmonização do contributo dos parceiros, evidente na sincronia e contingência dos comportamentos (Beeghly, et al., 2011). Deste modo, o conceito de regulação emocional é entendido como um constructo diádico em que bebé e adulto respondem aos comportamentos e emoções um do outro, de forma a regular as interações momento-a-momento, mutuamente (Tronick, 2007; Beeghly, et al., 2011).

Na esteira de definição da sensibilidade materna como um constructo diádico que inclui a resposta materna e infantil, mas igualmente, a relação estabelecida (contingência, sincronia e jogo) recorremos a medidas que incluem esta perspetiva para estudar e comparar a relação com bebés transportados em meios auxiliares ou junto ao corpo da mãe.

\section{Presente Estudo}

Sabendo que a forma como os bebés são transportados pelas suas mães pode afetar a regularidade, continuidade e sincronia das interações, temos como objetivo geral estudar e comparar a qualidade da 
relação nas díades portuguesas em que as mães transportam as filhas ou filhos junto ao corpo e nas díades em que as mães que transportam os bebés por meios auxiliares de transporte. Como objetivos específicos, pretendemos: (i) comparar a sensibilidade materna e a cooperação infantil em díades com transporte corporal e em díades com transporte por meios auxiliares; e (ii) comparar os dois grupos quanto à qualidade da resposta facial, resposta vocal, afetividade, contingência, atividade lúdica e reciprocidade, tanto nas mães como nos bebés.

Com base na revisão de literatura, prevemos que a proximidade física beneficie a interação mãefilho(a), por essa razão, hipotetizamos que as médias de sensibilidade materna e cooperação infantil sejam superiores no grupo dos bebés com transporte corporal comparativamente ao outro grupo.

\section{MÉTODO}

\section{Participantes}

Para concretizar os objetivos de estudo foi analisado o comportamento interativo de 40 díades mãe-filho(a) em jogo livre. Participaram 20 mães que transportavam os seus bebés preferencialmente junto ao seu corpo e na zona do peito (método babywearing), em comparação com 20 com mães que transportavam os seus bebés, sobretudo, através de carrinhos ou outros meios auxiliares.

Os dados demográficos que descrevem os bebés e as suas mães encontram-se na Tabela 1. Procurámos emparelhar as amostras em termos de idade gestacional (com um erro máximo de uma semana - inferior/superior), peso gestacional (com um erro inferior/superior até 100g), por idade da criança (com um erro inferior/superior até 2 meses), por idades dos pais (com um erro inferior/superior até 2 anos), nível socioeconómico, e nacionalidade (todas e todos os participantes são portuguesas/es). Não foi possível emparelhar os participantes por escolaridade das mães. Demonstrando a equivalência das amostras nestes critérios, à exceção da escolaridade materna, nenhum dos fatores apresentados na Tabela 1 são significativamente diferentes nos dois grupos de estudo.

Tabela 1. Médias e Desvio Padrão das variáveis demográficas

\begin{tabular}{|c|c|c|c|c|c|c|}
\hline & \multicolumn{4}{|c|}{ Grupo de Estudo } & \multirow{3}{*}{$t$} & \multirow{3}{*}{$p$} \\
\hline & \multicolumn{2}{|c|}{ TC } & \multicolumn{2}{|c|}{ TMA } & & \\
\hline & $M$ & $D P$ & $M$ & $D P$ & & \\
\hline Idade da criança (meses) & 17.85 & 8.34 & 16.15 & 4.95 & .783 & .44 \\
\hline Idade do adulto (anos) & 34.55 & 3.75 & 33.25 & 5.0 & .930 & .36 \\
\hline Apgar ao $1 .^{\circ}$ minuto & 8.82 & 1.66 & 8.80 & 1.28 & .034 & .97 \\
\hline Apgar ao $5 . \stackrel{o}{ }$ minuto & 9.67 & 1.05 & 9.80 & .41 & -.761 & .45 \\
\hline Idade gestacional do bebé & 39.20 & .95 & 39.44 & 1.00 & -.835 & .41 \\
\hline $\begin{array}{l}\text { Peso do bebé à nascença em } \\
\text { gramas }\end{array}$ & 3172.89 & 434.75 & 3281.70 & 377.88 & -.521 & .67 \\
\hline Escolaridade da Mãe & 16.30 & 1.75 & 13.75 & 2.53 & 3.706 & .001 \\
\hline Número de irmãos & .75 & .72 & 1.20 & 1.00 & -1.630 & .11 \\
\hline
\end{tabular}

As mães praticantes de transporte corporal indicaram que não o fizeram por necessidade financeira ou por questões de saúde, mas foi uma escolha essencialmente por motivações associadas a convicções, conveniência e preocupações parentais. Numa sociedade, em que norma é transportar os bebés em carrinhos, especialmente fora de casa, as famílias fazem-no sobretudo por motivos de segurança, proteção e facilidade.

\section{Instrumentos e procedimento}

Aos participantes foram apresentados os objetivos e procedimentos do estudo e entregue um documento explicativo. Todas as mães participantes neste estudo, deram o seu consentimento informado para a sua participação. Neste documento garantiu-se a confidencialidade e a desistência sem qualquer prejuízo para as famílias em qualquer momento do estudo.

O levantamento de dados demográficos, clínicos e de desenvolvimento foi realizado através de fichas de anamnese dirigida aos pais. Depois desta recolha de dados pediu-se às mães que brincassem com os bebés espontaneamente, tal como faziam habitualmente. Com efeito, as díades foram filmadas em situação de jogo livre durante 5 minutos seguindo o protocolo proposto por Crittenden (2003). As filmagens decorreram em espaços do quotidiano das famílias. 
Os registos videográficos foram observados pelas autoras do estudo que, para avaliar a qualidade de interação mãe-filho(a), usaram duas escalas: A CARE-Index (Crittenden, 2003) e a MINDS (Fuertes et al., 2014). Da escala internacional Care-Index utilizámos as pontuações globais da Sensibilidade Materna e da Cooperação Infantil para conhecer a qualidade geral da relação mãe-filho(a), tendo deste modo uma medida comparativa com estudos internacionais. A partir da escala MINDS recorremos à cotação das subescalas: resposta facial; resposta verbal; resposta afetiva; reciprocidade; contingência e qualidade de jogo cujos itens foram definidos a partir de observações nacionais e, portanto, mais sensíveis às singularidades das díades portuguesas.

Child-Adult Relationship Experimental (CARE-Index). A escala Care-Index desenvolvida por Patricia Crittenden (2003) avalia a sensibilidade, controlo e passividade materna bem como a cooperação, complusão/submissão, dificuldade e passividade infantil. Cada uma destas escalas é pontuada tendo em conta o comportamento dos dois parceiros em termos de interação facial, vocal, posicionamento/toque, afetividade, contingência, reciprocidade e qualidade de jogo. Crittenden, numa perspetiva diádica da sensibilidade materna, considera o comportamento em relação ao comportamento infantil e vice-versa. Por exemplo, a expressão facial da mãe pode pontuar como sensível, como controladora ou passiva. As escalas quantificam o padrão de resposta do adulto, repartindo entre elas, um total de 14 pontos. A escala inclui uma pontuação final da sensibilidade materna e cooperação infantil que indica a qualidade da relação observada durante a interação, medidas estas que foram utilizadas no nosso estudo.

De acordo com as instruções do manual de avaliação da escala CARE-Index, cada díade foi filmada durante cinco minutos. Antes da filmagem, foi pedido às mães para interagirem com as crianças espontaneamente e de forma natural, utilizando os brinquedos que desejassem e como normalmente faziam.

Mother-Infant Descriptive Dyadic System (MINDS). Na escala Mother-Infant Descriptive Dyadic System (MINDS, Fuertes, et al., 2014) analisam-se aspetos particulares da qualidade da interação. Observa-se detalhadamente a sincronia e a reciprocidade diádica, o grau de participação e envolvimento dos dois parceiros, o ambiente de interação, a qualidade das respostas afetivas, vocais e faciais (e.g. contacto ocular, comunicação, proximidade), e a capacidade do adulto oferecer um jogo adequado para idade e desenvolvimento da criança. A presente escala é uma adaptação portuguesa da escala CARE-Index que teve início num estudo com 200 díades portuguesas (Fuertes, Faria, Soares, \& Oliveira-Costa, 2010). O objetivo, partindo dos constructos e escalas da CARE-Index, pretendeu desenvolver indicadores com base nas observações portuguesas. Posteriormente, o estudo da validade da MINDS foi alargado a 400 díades (Serradas, Tadeu, Soares, Fuertes, 2016), permitindo incluir os comportamentos maternos e infantis mais frequentes e mais atípicos na população portuguesa.

A MINDS apresenta uma pontuação de 35 pontos (que corresponde à sensibilidade máxima em todas as áreas avaliadas) igualmente distribuída por 5 áreas: resposta facial, resposta vocal, trocas afetivas, posicionamento e manipulação, contingência, atividade lúdica e reciprocidade. A escala é pontuada de 1 a 5 em cada uma destas dimensões.

A pontuação 5 corresponde a interações de excelente qualidade (momentos de genuína alegria, prazer na companhia no outro, sincronia). A pontuação 4 corresponde a interações adequadas, mas com momentos de dissonância. A pontuação 3 corresponde a interações com problemas, mas sem risco para a criança (momentos de controlo ou passividade materna, mas com atenção ou atitude serena acompanhada de momentos de dificuldade, passividade ou compulsão infantil). A pontuação 2 corresponde a comportamentos infantis difíceis na sua relação com adultos passivos ou ambivalentes no seu comportamento, combinando passividade e contingência (e.g. pouca expressão afetiva, diretivos no jogo com a criança e vice-versa). A pontuação 1 corresponde a interações de risco, punitivas ou sem participação dos pares.

Acordo de cotadores. Cada cotador fez uma cotação independente, e o nível de acordo calculado a partir do coeficiente alfa de Cronbach foi superior a .81 para as duas escalas; as diferenças de pontuação foram resolvidas em conferência de cotadores.

\section{Tratamento dos dados}

Os dados foram analisados com recurso a estatística descritiva e inferencial usando a versão 22 do programa SPSS. Para optar por testes paramétricos ou não paramétricos, a normalidade da distribuição das variáveis foi testada. A estatística descritiva foi usada para calcular as médias e os respetivos desvios padrão dos dados demográficos. A estatística inferencial foi usada para calcular as diferenças de médias entre os dois grupos de estudo através do teste $t$-student. 0 nível de significância foi assumido a 05 . 


\section{RESULTADOS}

\section{Comparação da qualidade interativa em díades com transporte corporal e em díades com transporte por meios auxiliares}

O primeiro objetivo deste estudo era comparar a qualidade interativa em díades com transporte corporal e em díades com transporte por meios auxiliares. Para o efeito, comparámos as médias das pontuações da escala Care-Index através do teste $t$-student para amostras independentes. Os resultados indicaram uma diferença significativa entre os dois grupos de estudo, sendo que as mães transportaram os bebés corporalmente eram significativamente menos sensíveis do que as mães que utilizavam outros meios de transporte (Tabela 2). 0 presente estudo indicou, igualmente, que os bebés transportados junto ao corpo das mães eram significativamente menos cooperativos do que os bebés transportados por meios auxiliares (Tabela 2)

Tabela 2. Médias, desvio padrão e teste de diferença de médias na sensibilidade materna e cooperação do bebé no grupo dos bebés transportados corporalmente (TC) e no grupo dos bebés transportados por outros meios auxiliares (TMA)

\begin{tabular}{|c|c|c|c|c|c|c|}
\hline & \multicolumn{4}{|c|}{ Grupo de Estudo } & \multirow{3}{*}{$t$} & \multirow{3}{*}{$p$} \\
\hline & \multicolumn{2}{|c|}{ TC } & \multicolumn{2}{|c|}{ TMA } & & \\
\hline & $M$ & $D P$ & $M$ & $D P$ & & \\
\hline Sensibilidade Materna & 8.45 & 2.35 & 10.15 & 2.52 & -2.207 & .033 \\
\hline Cooperação Infantil & 8.30 & 2.51 & 10.20 & 2.36 & -2.467 & .018 \\
\hline
\end{tabular}

\section{Comparação das respostas, vocais, afetivas, diretivas, lúdicas e recíprocas em díades com transporte corporal e em díades com transporte por meios auxiliares}

Num segundo objetivo, quisemos comparar as respostas: vocais, afetivas, diretivas, lúdicas e recíprocas em díades com transporte corporal e em díades com transporte por meios auxiliares, tanto nas mães (Tabela 3) como nos bebés (Tabela 4). Os resultados, obtidos com a escala MINDS, indicaram que as mães que transportaram os bebés por meios auxiliares apresentam pontuações significativamente superiores, e portanto indicadoras de melhor qualidade, quanto à contingência e de reciprocidade do que as mães que transportavam os bebés corporalmente (Tabela 3).

Tabela 3. Médias, desvio padrão e teste de diferença de médias na qualidade das respostas faciais, vocais, afetivas, contingência, atividade lúdica e reciprocidade em mães que usaram o transporte corporal (TC) e em mães que usaram outros meios auxiliares de transporte (TMA)

\begin{tabular}{|c|c|c|c|c|c|c|}
\hline & \multicolumn{4}{|c|}{ Grupo de Estudo } & \multirow{3}{*}{$t$} & \multirow{3}{*}{$p$} \\
\hline & \multicolumn{2}{|c|}{ TC } & \multicolumn{2}{|c|}{ TMA } & & \\
\hline & $M$ & $D P$ & $M$ & $D P$ & & \\
\hline Respostas faciais & 3.45 & .94 & 4.00 & .97 & -1.814 & .078 \\
\hline Respostas vocais & 3.50 & 1.23 & 3.45 & 1.43 & .118 & .906 \\
\hline Respostas afetivas & 3.55 & 1.10 & 3.90 & 1.07 & -1.020 & .314 \\
\hline Contingência & 2.75 & 1.16 & 3.55 & 1.05 & -2.282 & .028 \\
\hline Atividade lúdica & 3.35 & 1.37 & 3.65 & .99 & -.788 & .436 \\
\hline Reciprocidade & 2.75 & 1.45 & 3.95 & .89 & -3.163 & .003 \\
\hline
\end{tabular}

Do lado dos bebés, verifica-se que aqueles que são transportados por meios auxiliares apresentavam pontuações significativamente superiores, em termos de respostas vocais, afetivas e de reciprocidade, comparativamente aos bebés do outro grupo de estudo (tabela 4). 
Tabela 4. Médias, desvio padrão e teste de diferença de médias na qualidade das respostas faciais, vocais, afetivas, contingência, atividade lúdica e reciprocidade em bebés transportados corporalmente pelas suas mães (TC) e em bebés transportados por outros meios auxiliares pelas suas mães (TMA)

\begin{tabular}{|c|c|c|c|c|c|c|}
\hline & \multicolumn{4}{|c|}{ Grupo de Estudo } & \multirow{3}{*}{$t$} & \multirow{3}{*}{$p$} \\
\hline & \multicolumn{2}{|c|}{ TC } & \multicolumn{2}{|c|}{ TMA } & & \\
\hline & $M$ & $D P$ & $M$ & $D P$ & & \\
\hline Respostas faciais & 3.20 & 1.28 & 3.70 & .87 & -1.447 & .156 \\
\hline Respostas vocais & 2.85 & 1.31 & 4.05 & 1.05 & -3.198 & .003 \\
\hline Respostas afetivas & 3.05 & 1.10 & 3.80 & 1.15 & -2.107 & .042 \\
\hline Contingência & 2.95 & 1.10 & 3.60 & .99 & -1.961 & .057 \\
\hline Atividade lúdica & 3.55 & 1.32 & 4.00 & 1.17 & -1.143 & .260 \\
\hline Reciprocidade & 2.50 & 1.43 & 3.90 & .97 & -3.621 & .001 \\
\hline
\end{tabular}

\section{DISCUSSÃO}

O presente estudo procurou comparar, numa situação de jogo livre, o comportamento interativo das mães que transportam os seus bebés junto ao corpo (zona do peito) e das mães que utilizam outras formas de transporte dos seus bebés. Pretendia-se analisar a qualidade interativa das mães quanto à resposta facial, resposta vocal, afetividade, contingência, atividade lúdica e reciprocidade nos dois grupos de estudo.

Quando comparámos os comportamentos interativos das díades, os resultados indicaram que as mães que transportavam os filhos junto ao corpo eram menos sensíveis do que as outras mães em estudo (que utilizavam outros meios auxiliares de transporte). Por seu lado, os bebés transportados junto ao corpo das mães nos primeiros meses de vida apresentam menor frequência e intensidade de comportamentos cooperativos.

Os resultados surpreenderam-nos na medida em que era plausível, à luz dos estudos prévios, que a proximidade física pudesse favorecer a relação materna. Com efeito, num estudo com mães oriundas de famílias socioeconomicamente desfavorecidas verificou-se uma prevalência superior de vinculação segura em bebés transportados corporalmente (Anisfeld, et al., 1990). Não obstante, a nossa amostra tinha um elevado nível de literacia considerando os níveis educacionais da população portuguesa. Ora, a primeira questão colocada é se este transporte poderá ser mais útil para algumas famílias. A investigação tem indicado que a vinculação e o estabelecimento das relações dependem das condições socioeconómicas das famílias e as respostas devem ser diferenciadas de acordo com as suas necessidades (Black et al., 2017).

Quando analisamos com mais detalhe os estudos anteriores verificamos que nestes estudos, realizados nos EUA, os bebés transportados em carrinhos são colocados virados para a frente e de costas para as mães. Não temos dados portugueses, mas uma observação cotidiana indica-nos que em Portugal, a maioria das mães, leva os bebés virado para si nos primeiros meses de vida. Ora, sendo a pesquisa tão escassa é impossível descortinar o impacto deste aspeto nos resultados.

0 transporte corporal dos bebés deve remontar aos tempos em que a humanidade era nómada como necessidade de se deslocar sem os mecanismos auxiliares atuais. Contudo, as mães do nosso estudo recorrem a versões ocidentalizadas deste tipo de transporte, sem contexto ou tradição cultural. Assim, os nossos dados devem ser analisados com cautela. Não se exclui a importância do transporte corporal, os dados devem ser interpretados à luz do contexto nacional e das condições de recolha. Com efeito, com a ocidentalização do conceito surgiram novas formas e razões de praticar babywearing. As mães praticantes de transporte corporal, no nosso estudo, indicam que não o fizeram por necessidade, mas sim por motivações associadas a convicções, conveniência e preocupações parentais. Estas motivações podem ter impacto nos resultados.

De um modo geral, os nossos resultados indicam que a proximidade física por si só, não é suficiente para promover boas interações mãe-filho(a), é necessário que as díades apresentem reciprocidade, qualidade afetiva e de jogo (Feber et al., 2008). Ora, aqui levantamos a questão se as mães portuguesas conseguem, como noutras culturas, já adicionar a experiência de transporte corporal com a atenção e resposta às solicitações dos bebés. De facto, quando comparamos expressão facial, expressão vocal, afetividade, reciprocidade, contingência e atividade lúdica nos dois grupos de estudos, verificamos que as mães do nosso estudo que transportam corporalmente os seus filhos apresentam piores indicadores na reciprocidade e contingência. Ora, o nosso estudo levanta uma nova questão de estudo: será que estas mães já apresentavam problemas de interação com os filhos e a opção pelo babywearing foi uma tentativa 
de reparação? Ou não tendo a experiência culturalmente transmitida do uso do pano, será que as mães portuguesas usaram corretamente o babywearing ou a forma como usaram inibiu interações com sincronia e reciprocidade?

Assim, os nossos dados parecem apoiar as perspetivas de que a vinculação e a relação-mãe decorrem de um processo adaptativo não meramente relacional mas também cultural (Belsky, 1999; Crittenden, 2000; Simpson, 1999).

Numa sociedade habituada ao transporte corporal, os tempos e as rotinas podem ser distintas daquelas que observamos neste estudo. Efetivamente, a maioria das mães do nosso estudo trabalhava e tinha mais filhos, por isso, passa a maior parte do dia fisicamente longe deles. Porventura, a reparação da separação diária carece de atividades que estimulem as trocas interativas que contribuem para a confiança mútua, envolvimento e satisfação (Kochanska, 1997; Tarabulsy et al., 2005).

Em suma, os nossos resultados corroboram a hipótese de que a proximidade física tem de resultar de interações envolventes e prazerosas e não meramente como forma de transporte já anteriormente aduzida por Tronick (1995). Por outro lado, os aspetos culturais podem ter um peso importante que ainda importa explorar. Sameroff e Fiese (2000) postularam que a qualidade interativa diádica decorre de fatores individuais, interacionais e sociais - como membros de uma comunidade com as suas redes sociais, normas, valores e atitudes próprias - e é influenciada pelos seus diferentes contextos e as suas inter-relações. No caso da vinculação, está bem documentado que a cultura afeta a qualidade da vinculação mãe-filho(a) (revisão em Faria, Lopes-dos-Santos, Fuertes, 2014; van IJzendoorn, \& SagiSchwartz, 2008). Ora, o contributo sóciocultural (numa abordagem bioecológica) da vinculação poderá ajudar a compreender os resultados na investigação sobre o papel do contacto e transporte corporal na relação mãe-filho.

Com base nos nossos resultados, e na esteira da investigação anterior, sugerimos que as intervenções na vinculação se devam centrar na interação diádica: promovendo a sensibilidade dos Pais (mães e pais), a comunicação entre Pais e crianças e a interação recíproca entre eles (Fuertes 2011; Fuertes \& Luis, 2014; Fuertes \& Santos, 2015).

\section{Contributos, limitações do estudo e perspetivas futuras}

0 presente estudo apresenta limitações próprias dos trabalhos experimentais, como por exemplo, o comportamento dos participantes pode ser influenciado pelo efeito da filmagem. Não obstante, e apesar do reduzido número de participantes, que impede a generalização dos resultados, julgamos ter contribuído para a reflexão sobre a importância de algumas variáveis maternas e infantis na organização dos processos da vinculação. Neste caso, ao contrário do esperado, a proximidade corporal não está associada ao aumento da sensibilidade diádica. Assim, os resultados desta pesquisa desafiam-nos a pensar sobre o real impacto da proximidade física na relação mãe-bebé e no contexto cultural em que essa proximidade ocorre.

Futuramente, procuraremos aumentar a dimensão da amostra e incluir participantes com maior diversidade cultural incluindo famílias de diversas culturas que tradicionalmente usam o modelo de transporte corporal. Por outro lado, é nossa intenção analisar as interações com transporte corporal de modo distinto, diferenciando o transporte junto ao peito que permite a interação face a face e o transporte às costas.

\section{REFERÊNCIAS}

Ainsworth, M. D., Blehar, M., Waters, E., \& Wall, S. (1978). Patterns of attachment- A psychological study of the Strange Situation. New Jersey, NJ, US: Lawrence Eribaum Associates.

Ainsworth, M., Bell, S. M., \& Stayton, D. J. (1974). Infant-mother attachment and social development: Socialisation' as product of reciprocal responsiveness to signals. In: M. Richards (ed.). The integration of a child into a social world (pp. 9-135). London, UK: Cambridge University Press.

Anisfeld, E., Casper, V., Nozyce, M., \& Cunningham, N. (1990). Does infant carrying promote attachement? An experimental study of the effects of increased physical contacto on the development of attachment. Child Development, 61, 1617-1627. http://dx.doi.org/10.1111/j.14678624.1990.tb02888.x

Beeghly, M., Fuertes, M., Liu, C., Delonis, M., \& Tronick, E. (2011). Maternal sensitivity in dyadic context: mutual regulation, meaning-making, and reparation. In D. W. Davis \& M. C. Logsdon (eds.), Maternal sensitivity: a scientific foundation for practice (pp. 45-69). Hauppauge, NY: Nova science publishers.

Belsky, J. (1999). Modern evolutionary theory and patterns of attachment. In J. Cassidy \& P. R. Shaver (Eds.), Handbook of attachment: Theory, research, and clinical applications (pp. 141-161). New York, NY, US: Guilford Press. 
Bernier, A., Carlson, S. M., \& Whipple, N. (2010). From external regulation to self-regulation: Early parenting precursors of young children's executive functioning. Child Development, 81, 326-339. http://dx.doi.org/10.1111/j.1467-8624.2009.01397.x

Black, M. et al. (2007). Advancing Early Childhood Development: from Science to Scale 1: Early childhood development coming of age: science through the life course. Lancet, 389(10064): 77-90. http://dx.doi.org/10.1016/S0140-6736(16)31389-7.

Blois, M. (2005). Babywearing: The Benefits and Beauty of This Ancient Tradition. US, Texas: Praeclarus.

Bowlby, J. (1969/1982). Attachment and loss (Vol. I). London: Penguin Book.

Calkins, S. D., \& Hill, A. (2007). Caregiver influences on emerging emotion regulation. In J. Gross (Ed.), Handbook of Emotion Regulation (pp. 229-248). New York, NY, US: Guilford Press.

Cassidy, J. (2008). The nature of the child's ties. In J. Cassidy \& P. R. Shaver (Eds.), Handbook of attachment: Theory, research, and clinical applications (pp. 3-22). New York, NY, US: Guilford Press.

Crittenden, P. M. (1988). Relationships at risk. In J. Belsky \& T. Nezworski (Eds.) The clinical implications of attachment. Hillsdale, NJ, US.: Lawrence Erlbaum.

Crittenden, P. M. (1999). A dynamic-maturational approach to continuity and change in pattern of attachment. In J. I. Vondra \& D. Barnett (Eds.), Atypical attachment in infancy and early childhood among at developmental risk. Monographs of the society for research in Child Development, 258(64), $145-171$.

Crittenden, P. M. (2000). A dynamic-maturational exploration of the meaning of security and adaptation: Empirical, cultural, and theoretical considerations. In P. M. Crittenden \& A. H. Claussen (eds). The organization of attachment relationships: Maturation, culture, and context (pp. 358-384). New York, NY, US: Cambridge University Press.

Crittenden, P. M. (2003). CARE-Index Manual (não publicado). Miami: IASA.

Crockenberg, S. C., Leerkes, E. M., \& Barrig Jo, P. S. (2008). Predicting aggressive behavior in the third year from infant reactivity and regulation as moderated by maternal behavior. Development and Psychopathology, 20, 37-54. http://dx.doi.org/10.1017/S0954579408000023

De Wolff, M. S., \& van IJzendoorn, M. H. (1997). Sensitivity and attachment: A Meta-Analysis on Parental Antecedents of Infant Attachment. Child Development, 68(4), 571-591. http://dx.doi.org/10.2307/1132107.

Faria, A., Lopes-dos-Santos, P., \& Fuertes, M., (2014). Pais e mães protegem, acarinham e brincam de formas diferentes. Análise Psicológica, 4(XXXII), 419-437. http://dx.doi.org/10.14417/ap.698

Ferber, S. G., Feldman, R., \& Makhoul, I. R. (2008). The development of maternal touch across the first year $\begin{array}{llll}\text { of } \quad \text { life. Early Human } & \text { Bevelopment, }\end{array}$ http://dx.doi.org/10.1016/j.earlhumdev.2007.09.019

Fuertes, A. Faria, A, Soares, H., \& Oliveira-Costa, A. (2010). Momentos de interação em que as emoções apre(e)ndem: estudo exploratório sobre a prestação materna e infantil em jogo livre. Psicologia USP, 21, 4, 833-857. http://dx.doi.org/10.1590/S0103-65642010000400010

Fuertes, M. \& Luís, H. (2014). Vinculação, práticas educativas na primeira infância e intervenção precoce. Revista Interacções, 30, 10, 1-7.

Fuertes, M. \& Santos, M. (2015). Parenting and Attachment in Portuguese Families. In G. Nicolas, A. Bejarano, \& D. L. Lee. Contemporary Parenting: A Global Perspective (pp. 156-171). London, UK: Routledge Press.

Fuertes, M. (2011). Intervenção Precoce: em que perspectiva? In T. Vasconcelos, Conselho Nacional das crianças dos 0 aos 3 anos, (pp. 117-120). Lisboa: CNE.

Fuertes, M., Canelhas, D., Oliveira-Costa, A., Faria, A., Ribeiro, C., Soares, H., \& Lopes dos Santos, P. (2014). Mother-infant descriptive dyadic system - MINDS.

Fuertes, M., Justo, M., Barbosa, M., Leopoldo, L., Lopes, J., Gomes Pedro, J. \& Sparrow, J. (2012). Infants prematurely born: Socio-emotional Development and Early Intervention. Diogo Contreiras and Johann Sampaio (Eds.). In Preterm Infants: Development, Prognosis and Potential Complications (pp. 100-125). NY: Nova Science Publishers, Inc.

Herrera, E., Reissland, N. \& Shepherd, J. (2004). Maternal touch and maternal child-directed speech: effects of depressed mood in the postnatal period. Journal of affective disorders, 81 (1), 29-39. http://dx.doi.org/10.1016/j.jad.2003.07.001

Hunziker, U. A., \& Barr, R. G. (1986). Increased carrying reduces infant crying: a randomized controlled trial. Pediatrics, 77, 641-648.

Kochanska, G. (1997). Mutually responsive orientation between mothers and their young children: Implications for early socialization. Child Development, 68, 94-112. http://dx.doi.org/10.1111/1467-8721.00198 
Leerkes, E. M., Blankson, A. N., \& O'Brien, M. (2009). Differential effects of maternal sensitivity to infant distress and nondistress on social-emotional functioning. Child Development, 80, 762-775. http://dx.doi.org/10.1111/j.1467-8624.2009.01296.x

Lucassen, N., Tharner, A., Van IJzendoorn, M. H., Bakermans-Kranenburg, M. J., Volling, B. L., Verhulst, F. C., \& Tiemeier, H. (2011). The association between paternal sensitivity and infant father attachment security: A meta-analysis of three decades of research. Journal of Family Psychology, 25, 986-992. http://dx.doi.org/10.1037/a0025855.

Mireault, G. C., Rainville, B. S., \& Laughlin, B. (2018). Push or Carry? Pragmatic Opportunities for Language Development in Strollers vs. Backpacks. Infancy, 23(4):616-624, http://dx.doi.org/10.1111/infa.12238

Moore, G. A., Hill-Soderlund, A. L., Propper, C. B., Calkins, S. D., Mills-Koonce, W. R., \& Cox, M. J. (2009). Mother-infant vagal regulation in the Face-to-Face Still-Face paradigm is moderated by maternal sensitivity. Child Development, 80, 209-223. http://dx.doi.org/10.1111/j.1467-8624.2008.01255.x

Sameroff, A. \& Fiese, B. (2000). Transactional regulation and early intervention. In J.P. Shankoff, \& S. J. Meisels (Eds). Handbook as early childhood intervention (pp. 135-159). Cambridge: Cambridge University Press.

Serradas, A., Tadeu, B., Soares, H., \& Fuertes, M. (2016). Estudo da sensibilidade materna em díades de risco biológico, ambiental e acumulado. In Fuertes, M., Nunes, C., \& Rosa, J. (org). Evidências de Intervenção Precoce - CIED. (pp. 19-36). Lisboa: ESELX/IPL.

Simpson, J. A. (1999). Attachment theory in modern evolutionary perspective. In J. Cassidy \& P. R. Shaver (Eds.), Handbook of attachment: Theory, research, and clinical applications (pp. 115-140). New York, NY, US: Guilford Press.

Tamis-LeMonda, C. S., Bornstein, M. H., Baumwell, L., \& Damast, A. M. (1996). Sensitivity in parenting interactions across the first two years: Influences on children's language and play. Early Development and Parenting, 5, 173-183. http://dx.doi.org/10.1016/S0163-6383(96)90284-2

Tarabulsy, G. M., Bernier, A., Provost, M. A., Maranda, J., Larose, S., Moss, E., Larose, M. \& Tessier R. (2005). Another look inside the gap: Ecological contributions to the transmission of attachment in a sample of adolescent mother-infant dyads. Developmental Psychology, 41, 212- 224. http://dx.doi.org/10.1037/0012-1649.41.1.212

Tessier, R., Cristo, M., Velez, S., Giron, M., Calume, Z., Ruiz-Palaez, J., Charpak, Y., \& Charpak, N. (1998). Kangaroo mother care and the bonding hypothesis. Pediatrics, 102(2), 17. http://dx.doi.org/10.1542/peds.102.2.e17

Thompson, R. A. (2008). Early attachment and later development: Familiar questions, new answers. In J. Cassidy \& P. R. Shaver (Eds.), Handbook of attachment: Theory, research, and clinical applications (pp. 330-348). New York, NY, US: Guilford Press.

Tronick, E. Z. (1995). Touch in mother-infant interaction. In T. M. Field (Ed.), Touch in early development (pp. 53-65). Hillsdale, NJ, US: Lawrence Erlbaum Associates, Inc.

Tronick, E. Z. (2007). The neurobehavioral and social-emotional development of infants and children. New York, NY, US: Norton.

van IJzendoorn, M. H., \& Sagi-Schwartz, A. (2008). Cross-cultural patterns of attachment: Universal and contextual dimensions. In J. Cassidy \& P. R. Shaver (Eds.), Handbook of attachment: Theory, research, and clinical applications (pp. 880-905). New York, NY, US: Guilford Press.

Historial do artigo

Recebido $\quad 12 / 18$

Aceite $\quad 06 / 19$

Publicado 08/19 